\title{
Erratum to: Sharing Sustainability: How Values and Ethics Matter in Consumers' Adoption of Public Bicycle-Sharing Scheme
}

\author{
Juelin Yin $^{1} \cdot$ Lixian Qian ${ }^{1}$ Anusorn Singhapakdi ${ }^{2}$
}

Published online: 15 March 2016

(C) Springer Science+Business Media Dordrecht 2016

\section{Erratum to: J Bus Ethics DOI 10.1007/s10551-016-3043-8}

The full mail address of the first and second authors is: International Business School Suzhou, Xi'an JiaotongLiverpool University, No.111 Ren'ai Road, Suzhou Industrial Park, Suzhou 215123, Jiangsu, China

The authors of the above mentioned article would like to add the following paragraph:

Acknowledgments The authors thank the reviewers for their helpful comments. This research was supported by
Jiangsu Philosophy \& Social Science Funding Programme of Jiangsu Department of Education (Project No. 2015SJD618 and No. 2015SJD617) and National Natural Science Foundation of China (Grant No. 71573213, 71202025, 71402093). Authors also thank Professor Russell Belk, Professor Florian Kohlbacher and the seminar participants at $\mathrm{Xi}^{\prime}$ an Jiaotong-Liverpool University, for their helpful comments and suggestions on earlier versions of this article.
The online version of the original article can be found under doi:10.1007/s10551-016-3043-8.

Anusorn Singhapakdi

asinghap@odu.edu

Juelin Yin

yinjuelin@gmail.com

Lixian Qian

lixian.qian@xjtlu.edu.cn

1 International Business School Suzhou, Xi' an JiaotongLiverpool University, No.111 Ren'ai Road, Suzhou Industrial Park, Suzhou 215123, Jiangsu, China

2 Department of Marketing, Strome College of Business, Old Dominion University, Virginia, USA 